LA W RENCE LIVERMORE NATIONAL LABORATORY

Adaptive Wavenumber Estimation for Mode Tracking in a Shallow Ocean Environment

J. V. Candy

July 6, 2012

IEEE OCEANS ' 12

Hampton Roads, VA, United States

October 15, 2012 through October 18, 2012 
This document was prepared as an account of work sponsored by an agency of the United States government. Neither the United States government nor Lawrence Livermore National Security, LLC, nor any of their employees makes any warranty, expressed or implied, or assumes any legal liability or responsibility for the accuracy, completeness, or usefulness of any information, apparatus, product, or process disclosed, or represents that its use would not infringe privately owned rights. Reference herein to any specific commercial product, process, or service by trade name, trademark, manufacturer, or otherwise does not necessarily constitute or imply its endorsement, recommendation, or favoring by the United States government or Lawrence Livermore National Security, LLC. The views and opinions of authors expressed herein do not necessarily state or reflect those of the United States government or Lawrence Livermore National Security, LLC, and shall not be used for advertising or product endorsement purposes. 


\title{
Adaptive Wavenumber Estimation for Mode Tracking in a Shallow Ocean Environment
}

\author{
J. V. Candy, Fellow, IEEE
}

\begin{abstract}
The shallow ocean is an uncertain, varying, dispersive environment dominated by ambient and shipping noise as well as temperature fluctuations that alter sound propagation throughout creating a large number of environmental variations. The need to develop processors that are capable of tracking these changes while simultaneously providing enhanced signals implies a stochastic as well as an adaptive design is required. The stochastic requirement follows directly from the multitude of variations created by uncertain parameters and noise. An adaptive processor providing enhanced signal estimates for acoustic hydrophone measurements on a vertical array as well as enhanced modal function and wavenumber estimates is developed. A normal-mode model is transformed to state-space form and incorporated directly into the processor enabling the signal enhancement capabilities. Data synthesized from the wellknown Hudson Canyon experiment is used to demonstrate the viability of this approach.
\end{abstract}

Index Terms-adaptive model-based processor, sequential Bayesian processor, sequential Monte Carlo, particle filter, unscented Kalman filter

\section{INTRODUCTION}

The shallow ocean is a particularly challenging signal processing environment primarily because of its inherent dynamics created by temperature variations in the upper layers and both internal and external disturbances that directly alter the sound propagation throughout. Temperature variations directly impact sound speed due their strong interrelationship, while internal disturbances can be related to fish sounds (snapping shrimp, mammal communications). External disturbances are directly related to wind induced wave motion, shipping noise and other surface related noises. In all, the shallow ocean is quite a hostile environment to attempt to extract meaningful information from directly without sophisticated processing techniques.

Thus, the ocean is a dynamic, ever-changing environment that requires a processor that can adapt to these changes in a consistent manner. Adaptive processing can be achieved using a recursive or equivalently sequential formulation. Sequential processing enables the realization of such a processor in order to account for changes especially in a shallow ocean environment. The processor tracks these variations by adjusting parameters that are capable of capturing the environmental changes (nonstationary spatial/temporal variations) thereby mitigating them in the measured data and enhancing the signals of interest. Bayesian sequential processing incorporating propagation models along with their inherent environmental parameters as well as measurement and noise models offers a robust, parametrically adaptive solution to signal processing problems in such a nonstationary environment. Sequential
Bayesian techniques enable a class of processors capable of performing in an uncertain, nonstationary (varying statistics), non-Gaussian, variable shallow ocean environment. Here we address the problem of estimating or tracking modal functions in a hostile shallow ocean while jointly adjusting (adaptively) the inherent propagation model parameters (wavenumber).

Previous work on this problem has investigated adaptive solutions under Gaussian assumptions using approximate nonlinear processors such as extended Kalman filters with some success; however, here we attack the problem with the sequential Bayesian construct enabling the joint modal function and wavenumber estimation to proceed without any limiting statistical assumptions. It has already been shown that this parametrically adaptive approach can operate successfully in this environment [25], [26] when estimating modal coefficients; however, here we construct processors to adapt to the horizontal wavenumber-a more environmentally sensitive parameter. We begin by formulating the problem in a state-space framework and developing the necessary mathematics for processor design. Next a simulation is performed based on a wellknown ocean experiment in order to capture the environment and provide realistic data for the design. The performance of the Bayesian processor is analyzed indicating its capability to track both modes and wavenumber simultaneously while jointly enhancing the raw hydrophone measurements.

The basic approach we employ to solve this problem is model-based. Incorporating a propagation model into a signal processing scheme has evolved over a long period of time where it was recognized that by embedding a physics-based representation can significantly improve the processing [1][5]. In ocean acoustics there are many problems of interest [6]-[14] governed by propagation models of varying degrees of sophistication. Here we are interested in a shallow water environment characterized by a normal-mode model. The model-based approach offer a means of estimating various quantities of high interest, but it also provides a methodology to statistically evaluate its performance on-line [16].

In this paper, we are primarily interested in investigating the performance of the "next generation" of model-based signal processing algorithms, primarily the unscented Kalman filter $(U K F)$ and the particle filter $(P F)$ with the goal of analyzing their performance on pressure-field data synthesized from the well-known Hudson Canyon experiments performed on the New Jersey shelf [11], [12]. Recall that the $P F$ is a sequential Markov chain Monte Carlo (MCMC) Bayesian processor capable of providing reasonable performance for a multi-modal problem estimating a non-parametric representation of the 
posterior distribution [24]. We also compare the $P F$ with the $U K F$ which is a unimodal processor capable of representing any single peaked distribution using a statistical linearization technique based on sigma points that deterministically characterize the posterior [24].

Background for the state-space representation of our problem is given in Section II leading to the formulation of the forward propagator. The particular algorithms employed are discussed in the Appendix. The design of the $M B P$ for a shallow ocean acoustic problem is discussed in Section III and the results are given where we compare processor performance. We summarize and discuss our results in the final section.

\section{STATE-SPACE PROPAGATOR}

For our ocean acoustic signal enhancement problem we assume a horizontally-stratified ocean of depth $h$ with a known horizontal source range $r_{s}$ and depth $z_{s}$ and that the acoustic energy from a point source can be modeled as a trapped wave governed by the Helmholtz equation [9], [13]. The standard separation of variables technique and removing the time dependence leads to a set of ordinary differential equations, that is, we obtain a "depth only" representation of the wave equation which is an eigenvalue equation in $z$ with

$$
\frac{d^{2}}{d z^{2}} \phi_{m}(z)+\kappa_{z}^{2}(m) \phi_{m}(z)=0, m=1, \cdots, M
$$

whose eigensolutions $\left\{\phi_{m}(z)\right\}$ are the so called modal functions and $\kappa_{z}$ is the wavenumber in the z-direction. These solutions depend on the sound speed profile, $c(z)$, and the boundary conditions at the surface and bottom as well as the corresponding dispersion relation given by

$$
\kappa^{2}=\frac{\omega^{2}}{c^{2}(z)}=\kappa_{r}^{2}(m)+\kappa_{z}^{2}(m), \quad m=1, \ldots, M
$$

where $\kappa_{r}(m)$ is the horizontal wavenumber associated with the $m$-th mode in the $r$ direction and $\omega$ is the harmonic source frequency.

By assuming a known horizontal source range a priori, we obtain a range solution given by the Hankel function, $H_{0}\left(\kappa_{r} r_{s}\right)$ enabling the pressure-field to be represented by

$$
p\left(r_{s}, z\right)=\sum_{m=1}^{M} \beta_{m}\left(r_{s}, z_{s}\right) \phi_{m}(z)
$$

where $p$ is the acoustic pressure; $\phi_{m}$ is the $m^{t h}$ modal function with the modal coefficient defined by

$$
\beta_{m}\left(r_{s}, z_{s}\right):=q H_{0}\left(\kappa_{r} r_{s}\right) \phi_{m}\left(z_{s}\right)
$$

for $q$ is the source amplitude and $H_{0}$ is the zero-th Hankel function at horizontal wavenumber and source range $r_{s}$.

\section{A. State-Space Model}

The depth-only eigen-equation can easily be transformed to state-space form by defining the state vector of the $m$-th mode as

$$
\phi_{m}(z):=\left[\begin{array}{c}
\phi_{m}(z) \\
\frac{d}{d z} \phi_{m}(z)
\end{array}\right]=\left[\begin{array}{c}
\phi_{m 1}(z) \\
\phi_{m 2}(z)
\end{array}\right]
$$

Thus, we have for the $m$-th mode the following state (vector) equation as:

$$
\frac{d}{d z} \phi_{m}(z)=\mathbf{A}_{m}(z) \phi_{m}(z)
$$

for

$$
\mathbf{A}_{m}(z)=\left[\begin{array}{cc}
0 & 1 \\
-\kappa_{z}^{2}(m) & 0
\end{array}\right]
$$

Assuming that the ocean acoustic noise can be characterized by additive uncertainties, we can extend the deterministic state equation for the $M$-modes, that is, $\Phi(z):=$ $\left[\phi_{1}(z)|\cdots| \phi_{M}(z)\right]^{T}$ leading to the following $2 M$-dimensional Gauss-Markov representation of the model:

$$
\frac{d}{d z} \phi(z)=\mathbf{A}(z) \phi(z)+\mathbf{w}(z)
$$

where $\mathbf{w}(z)=\left[\begin{array}{llll}w_{1} & w_{2} & \ldots & w_{2 M}\end{array}\right]^{T}$ is additive, zero-mean random noise. The system matrix $\mathbf{A}(z)$ is defined as

$$
\mathbf{A}(z)=\left[\begin{array}{ccc}
\mathbf{A}_{1}(z) & \cdots & 0 \\
\vdots & \ddots & \vdots \\
0 & \cdots & \mathbf{A}_{M}(z)
\end{array}\right]
$$

and the overall state vector is

$$
\phi(z)=\left[\begin{array}{llllll|ll}
\phi_{11} & \phi_{12} \mid \phi_{21} & \phi_{22} \mid & \ldots & \phi_{M 1} & \phi_{M 2}
\end{array}\right]^{T}
$$

This representation leads to the measurement equations that we can write as

$$
p\left(r_{s}, z\right)=\mathbf{C}^{T}\left(r_{s}, z_{s}\right) \phi(z)+v(z)
$$

where

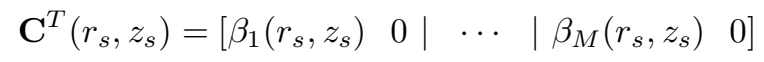

The random noise terms $\mathbf{w}(z)$ and $v(z)$ can be assumed Gaussian and zero-mean with respective covariance matrices, $\mathbf{R}_{w w}$ and $\mathbf{R}_{v v}$. The measurement noise $(v(z))$ can be used to represent the "lumped" effects of near-field acoustic noise field, flow noise on the hydrophone and electronic noise. The modal noise $(\mathbf{w}(z))$ can be used to represent the "lumped" uncertainty of sound speed errors, distant shipping noise, errors in the boundary conditions, sea state effects and ocean inhomogeneities that propagate through the ocean acoustic system dynamics (normal-mode model). These assumptions, with known model parameters lead to the optimal solution of the state estimation problem (Kalman filter) [18]. 
Since our array spatially samples the pressure-field discretizing depth, we choose to discretize the differential state equations using a central difference approach for improved numerical stability, that is, from Eq. 1 we have

$$
\frac{d^{2} \phi_{m}(z)}{d z^{2}} \approx \frac{\phi_{m}\left(z_{\ell}\right)-2 \phi_{m}\left(z_{\ell-1}\right)+\phi_{m}\left(z_{\ell-2}\right)}{\triangle z_{\ell}^{2}}
$$

for $\triangle z_{\ell}:=z_{\ell}-z_{\ell-1}$. Substituting this approximation into the modal relations of Eq. 1 gives

$\phi_{m}\left(z_{\ell}\right)-2 \phi_{m}\left(z_{\ell-1}\right)+\phi_{m}\left(z_{\ell-2}\right)+\triangle z_{\ell}^{2} \kappa_{z}^{2}(m) \phi_{m}\left(z_{\ell-1}\right)=0$

where $m=1, \cdots, M$ and $z_{\ell}$ is the location of the $\ell$-th sensor. Defining the discrete modal state vector as $\phi_{m}\left(z_{\ell}\right):=$ $\left[\phi_{m}\left(z_{\ell-2}\right) \mid \phi_{m}\left(z_{\ell-1}\right)\right]^{T}$, we obtain the following set of difference equations for the $m$-th mode

$$
\begin{aligned}
& \phi_{m 1}\left(z_{\ell}\right)=\phi_{m 2}\left(z_{\ell-1}\right) \\
& \phi_{m 2}\left(z_{\ell}\right)=-\phi_{m 1}\left(z_{\ell-1}\right)+\left(2-\triangle z_{\ell}^{2} \kappa_{z}^{2}(m)\right) \phi_{m 2}\left(z_{\ell-1}\right)
\end{aligned}
$$

with each of the corresponding modal $A$-submatrices given by

$$
\mathbf{A}_{m}(z)=\left[\begin{array}{cc}
0 & 1 \\
-1 & 2-\triangle z_{\ell}^{2} \kappa_{z}^{2}(m)
\end{array}\right] ; \quad m=1, \cdots, M
$$

\section{B. Parametrically Adaptive Processor}

The "parametrically adaptive" processor evolves from this representation by defining a parameter set of interest. Since we are primarily interested in an environmentally adaptive processor, that is, a processor capable of adjusting its parameters to variations in the environment such as temperature, noise, etc. We choose to capture these changes by allowing the horizontal wavenumber to vary as compared to our previous efforts [25], [26] that focused on modal coefficient estimation. Here, we define the parameter vector as

$$
\theta_{m}(z):=\kappa_{r}(m) ; m=1, \cdots, M
$$

and a new "augmented" state vector as

$$
\Phi_{m}\left(z_{\ell} ; \theta_{m}\right):=\Phi_{m}\left(z_{\ell}\right)=\left[\phi_{m 1}\left(z_{\ell}\right) \phi_{m 2}\left(z_{\ell}\right) \mid \theta_{m}\left(z_{\ell}\right)\right]^{T}
$$

With this choice of parameters (horizontal wavenumber) the augmented state equations for the $m$-th mode become

$$
\begin{aligned}
\phi_{m 1}\left(z_{\ell}\right) & =\phi_{m 2}\left(z_{\ell-1}\right)+w_{m 1}\left(z_{\ell-1}\right) \\
\phi_{m 2}\left(z_{\ell}\right) & =-\phi_{m 1}\left(z_{\ell-1}\right)+\left(2-\triangle z_{\ell}^{2}\left(\frac{\omega^{2}}{c^{2}\left(z_{\ell}\right)}-\theta_{m}^{2}\left(z_{\ell-1}\right)\right)\right) \\
& \times \phi_{m 2}\left(z_{\ell-1}\right)+w_{m 2}\left(z_{\ell-1}\right) \\
\theta_{m}\left(z_{\ell}\right) & =\theta_{m}\left(z_{\ell-1}\right)+w_{\theta_{m}}\left(z_{\ell-1}\right)
\end{aligned}
$$

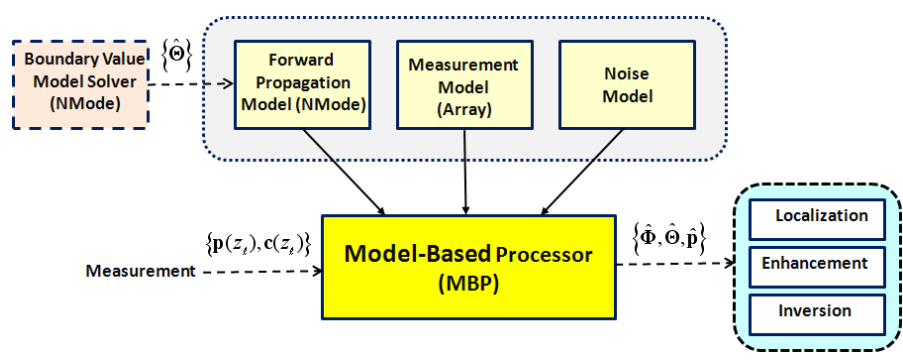

Fig. 1. Model-based processor design: (a) Boundary Solver for initial parameters. (b) Propagator, measurement and noise models. (c) MBP. (d) Applications: localization, enhancement (tracking) and inversion.

where we have selected a random walk model $\left(\dot{\theta}_{m}(z)=\right.$ $\left.w_{\theta_{m}}(z)\right)$ to capture the variations of the horizontal wavenumber with additive, zero-mean, Gaussian noise of covariance $R_{w_{\theta_{m}} w_{\theta_{m}}}$.

As mentioned previously [26] the random walk model can provide constraints in the simulation, since the parameter is modeled as Gauss-Markov implying that $95 \%$ of the samples must lie within confidence limits controlled by $\left( \pm 1.96 \sqrt{R_{w_{\theta_{m}} w_{\theta_{m}}}}\right)$. This constitutes a soft statistical constraint of the parameter variations [15]. For our runs, we choose to start the processor with initial parameter estimates close to those values other researchers have meticulously estimated from the Hudson Canyon data set [11], [12].

More succinctly, for the $m$-th mode we can write

$$
\Phi_{m}\left(z_{\ell}\right)=\mathbf{A}_{m}\left(z_{\ell-1}\right) \Phi_{m}\left(z_{\ell-1}\right)+\mathbf{w}_{m}\left(z_{\ell-1}\right)
$$

for

$\mathbf{A}_{m}\left(z_{\ell-1}\right)=\left[\begin{array}{cccc}0 & 1 & \mid & 0 \\ -1 & 2-\triangle z_{\ell}^{2}\left(\frac{\omega^{2}}{c^{2}\left(z_{\ell}\right)}-\theta_{m}^{2}\left(z_{\ell-1}\right)\right) & \mid & 0 \\ - & - & - & \\ 0 & 0 & \mid & 1\end{array}\right]$

The corresponding measurement model is given by

$p\left(r_{s}, z_{\ell}\right)=\sum_{m=1}^{M} \beta_{m}\left(r_{s}, z_{s} ; \theta_{m}\left(z_{\ell}\right)\right) \phi_{m}\left(z_{\ell}\right)+v\left(z_{\ell}\right) ; \ell=1, \cdots, L$

with

$$
\beta_{m}\left(r_{s}, z_{s}\right):=q H_{0}\left(\theta_{m}\left(z_{\ell}\right) r_{s}\right) \phi_{m}\left(z_{s}\right)
$$

This completes the section on the discrete state-space representation of the shallow ocean acoustic (normal-mode) propagation model that is embedded as a "forward propagator" into the subsequent processors for signal enhancement. Note that the initial model parameters are obtained from the prior (16) solution of the boundary value problem as shown in Fig. 1. 


\section{MODEL-BASED OCEAN ACOUSTIC PROCESSING}

In this section we discuss the development of the propagator for the Hudson Canyon experiment performed in 1988 in the Atlantic with the primary goal of investigating acoustic propagation (transmission and attenuation) using continuous wave data [11], [12]. The Hudson Canyon is located off the coast of New Jersey in the area of the Atlantic Margin Coring project borehole 6010 . The seismic and coring data are combined with sediment properties measured at that site. Excellent agreement was determined between the model and data indicating a well-known, well-documented shallow water experiment with bottom interaction and yielding ideal data sets for investigating the applicability of a $M B P$ to measured ocean acoustic data [11], [12]. The experiment was performed at low frequencies $(50-600 \mathrm{~Hz})$ in shallow water of $73 \mathrm{~m}$ depth during a period of calm sea state. A calibrated acoustic source was towed at roughly $36 \mathrm{~m}$ depth along the $73 \mathrm{~m}$ isobath radially to distances of 4 to $26 \mathrm{Km}$. The ship speed was between 2 and 4Kts. The fixed vertical hydrophone array consisted of 24 phones spaced $2.5 \mathrm{~m}$ apart extending from the sea-floor up to a depth of about $14 \mathrm{~m}$ below the surface. The normalized horizontal wavenumber spectrum for a $50 \mathrm{~Hz}$ temporal frequency is dominated by 5 modes occurring at wavenumbers between 0.14 to $0.21 \mathrm{~m}^{-1}$. A SNAP [6] simulation was performed and the results agree quite closely, indicating a well-understood ocean environment.

In order to construct the state-space propagator, we require the set of parameters which were obtained from the experimental measurements and processing (wavenumber spectra). The horizontal wavenumber spectra were estimated using synthetic aperture processing [11]. Eight temporal frequencies were employed: four on the inbounds $(75 \mathrm{~Hz}, 275 \mathrm{~Hz}, 575 \mathrm{~Hz}, 600 \mathrm{~Hz})$ and four on the outbound $(50 \mathrm{~Hz}, 175 \mathrm{~Hz}, 375 \mathrm{~Hz}, 425 \mathrm{~Hz})$. In this application we will confine our investigation to the $50 \mathrm{~Hz}$ case, which is well-documented, and to horizontal ranges from $0.5-4 \mathrm{Km}$. The raw measured data was processed (sampled, corrected, filtered, etc.) and supplied for this investigation.

\section{A. Adaptive PF Design}

The design and development of the environmentally adaptive $P F$ proceeds through the following steps as shown in Fig. 4: (1) pre-processing the raw experimental data; (2) solving the boundary value problem $(B V P)$ [9] to obtain initial parameter sets for each temporal frequency (e.g. wavenumbers, modal coefficients, initial conditions, etc.); (3) state-space forward propagator simulation of synthetic data for $P F$ analysis/design; (4) application to measured data; and (5) $P F$ performance analysis.

Pre-processing of the measured pressure-field data follows the usual pattern of filtering, outlier removal and Fourier transforming to obtain the complex pressure-field as a function of depth along the array. This data along with experimental conditions (frequencies, sound-speed profiles (CTD measurements), boundary conditions, horizontal wavenumber estimators (see [12] for details) provide the input to the normal mode $B V P$

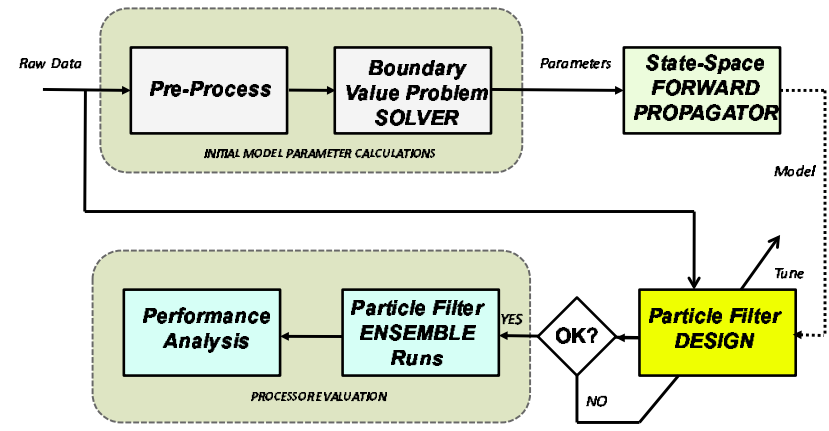

Fig. 2. $P F$ design/development procedure: (a) Initial parameters/conditions. (b) Design runs. (c) Ensemble runs.

solutions (SNAP [6], KRACKEN [7], etc.) yielding the output parameters. These parameters are then used as input to the state-space forward propagator (see Fig. 4) developed in Sec. II.

The state-space propagator is then used to develop a set of synthetic pressure-field data with higher resolution than the original raw data, that is, a 46-element array at halfwave inter-element spacing rather than the 23-element array used in the experiment. This set represents the "truth" data that can be investigated when "tuning" the $P F$ (e.g. number of particles, covariances, etc.). Once tuned, the processors are applied directly to the measured pressure-field data (23elements) after re-adjusting some of the processor parameters (covariances). Here the metrics are estimated and processor performance analyzed. Since each run of the $P F$ is a random realization, that is, the process noise inputs are random, an ensemble of results are estimated with ensemble statistics presented. In this way, we can achieve a detailed analysis of the processor performance prior to fielding and operational version. In this paper we constrain our discussion results to processing synthesized pressure-field measurements using a 46-element array.

\section{B. Results}

First we investigate the enhancement capabilities of the $P F$ in estimating the pressure-field over a 100 -member ensemble shown in Fig. 3. Using 1500-particles, we see the synthesized data (dashed blue line) as well as both maximum a-posteriori (MAP) estimates (red circles) and conditional mean $(C M)$ estimates (dotted magenta line with circles). Both estimators appear to track the field quite well (true (mean) solution in green dashes). The corresponding innovations (residual) sequence is also shown (black). Classically, both estimators produced satisfactory zero-mean/statistical whiteness tests (see Fig. 4 for $P F$ ) as well as the WSSR tests 


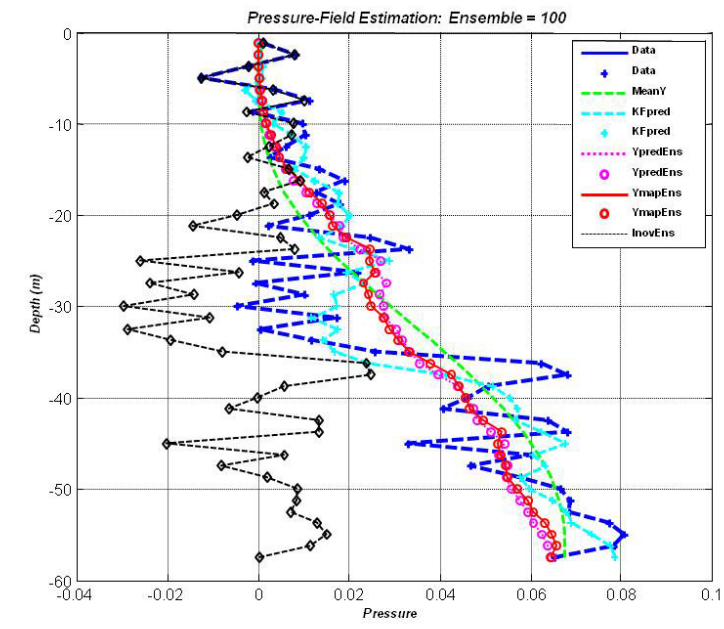

Fig. 3. Synthesized/enhanced pressure-field (blue dots) data from the Hudson Canyon experiment simulation with a 46-element hydrophone vertical array using particle filter estimators: MAP(red), conditional mean $(C M)$ in magenta and the $U K F$ (turquoise) with corresponding innovations (residuals) sequence (green).

indicating a "tuned" processor [18], that is, PF-(ZM-WT: $1.2 \times 10^{-4}<3.5 \times 10^{-1} / 3.13 \%$ out/WSSR below) and CM(ZM-WT: $1.58 \times 10^{-3}<3.5 \times 10^{-1} / 6.5 \%$ out/WSSR below). The UKF processor also produced reasonable results: UKF(ZM-WT: $1.3 \times 10^{-3}<3.5 \times 10^{-1} / 0.0 \%$ out/WSSR below) for the enhanced pressure-field.

Ensemble mode tracking results are shown in Figs. 5 and 6 for each of the modal function estimators, the $P F(M A P / C M)$ and the UKF. In Fig. 5 we observe that the performance of the $P F(M A P / C M)$ appears to track the modes quite well and slightly better than the UKF. The PF estimators perform equivalently. Two of the modal function estimates (first two) exhibit the largest errors while the final three functional estimates are much better. The root-mean-squared (modal tracking) error for each mode is quite reasonable: RMSE: $(12.1,7.6,14.2,1.9,8.8) \times 10^{-5}$ again confirming the difficulty the estimator is having to maintain track on the lower order modal functions. It is interesting to note that the wavenumber estimates are constantly being adapted (adjusted) by the processor throughout the runs attesting to the nonstationary nature of the ocean statistics as illustrated in Fig. 6. The ensemble average wavenumber estimates are very reasonable: (PF) 0.206, 0.197, 0.181, 0.173, 0.142; (CM) 0.206, 0.197, $0.181,0.173,0.141$; (TRUE) $0.208,0.199,0.183,0.175,0.142$. The $P F$ and $C M$ ensemble estimates are very close to the true values adapting to the changing ocean environment yet still preserving wavenumber values on the average. We summarize these reults in Table I. On a single realization, all three of three of the processors were capable of precisely predicting the correct values but the ensemble results give a better overall performance metric.

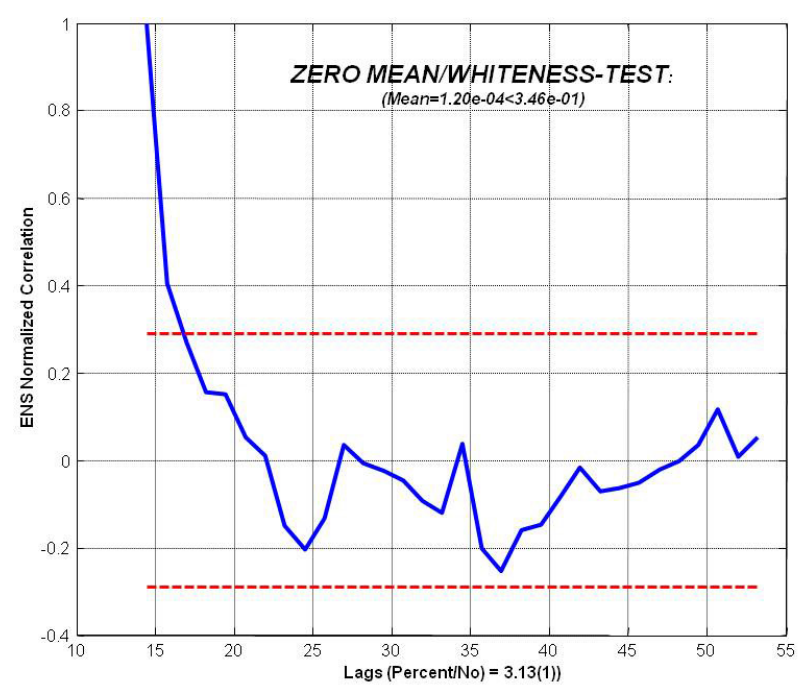

Fig. 4. Ensemble innovations zero-mean whiteness testing: $\left(1.2 \times 10^{-4}<\right.$ $3.5 \times 10^{-4}$ and $3.13 \%$ out)
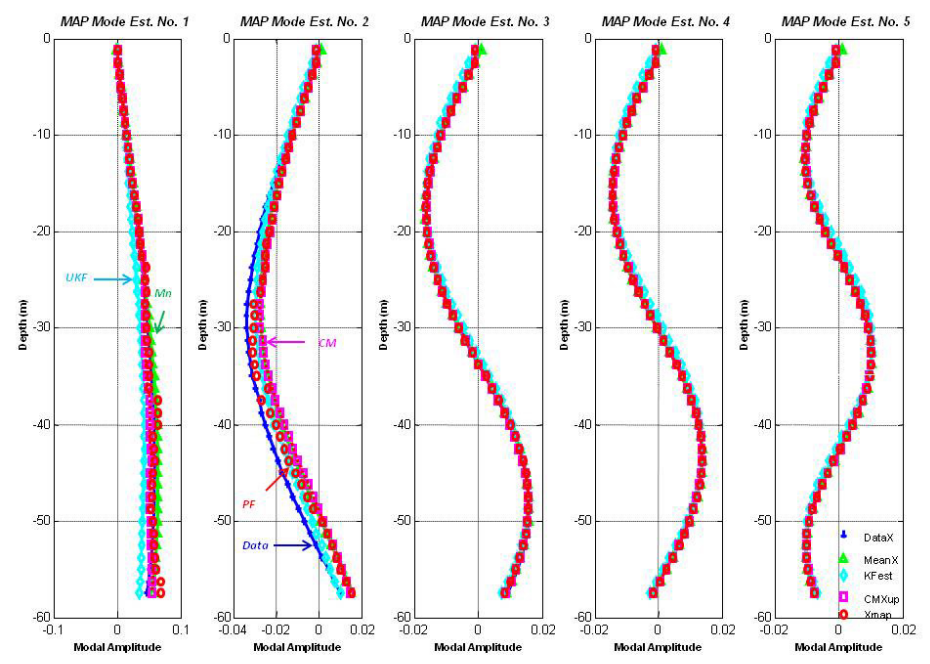

Fig. 5. Modal function tracking (estimation): synthesized Hudson Canyon data of a 46-element array (blue plus), UKF (turquoise dots), MAP (red circles) and $C M$ (magenta squares) particle filters.

Table I. Ensemble Wavenumber Estimation.

\begin{tabular}{||c|c|c|c||}
\hline \hline True & PF & CM & RMSE \\
\hline 0.208 & 0.206 & 0.206 & $12.1 \times 10^{-5}$ \\
\hline 0.199 & 0.197 & 0.197 & $7.6 \times 10^{-5}$ \\
\hline 0.183 & 0.181 & 0.181 & $14.2 \times 10^{-5}$ \\
\hline 0.175 & 0.173 & 0.173 & $1.9 \times 10^{-5}$ \\
\hline 0.142 & 0.142 & 0.141 & $8.8 \times 10^{-5}$ \\
\hline
\end{tabular}

We also illustrate the multimodal aspect of the oceanic data by observing the modal function posterior PDF estimates for modes 2 and 4 as illustrated in Fig. 7 and Fig. 8. It is clear from the plots that for each depth multiple peaks appear 

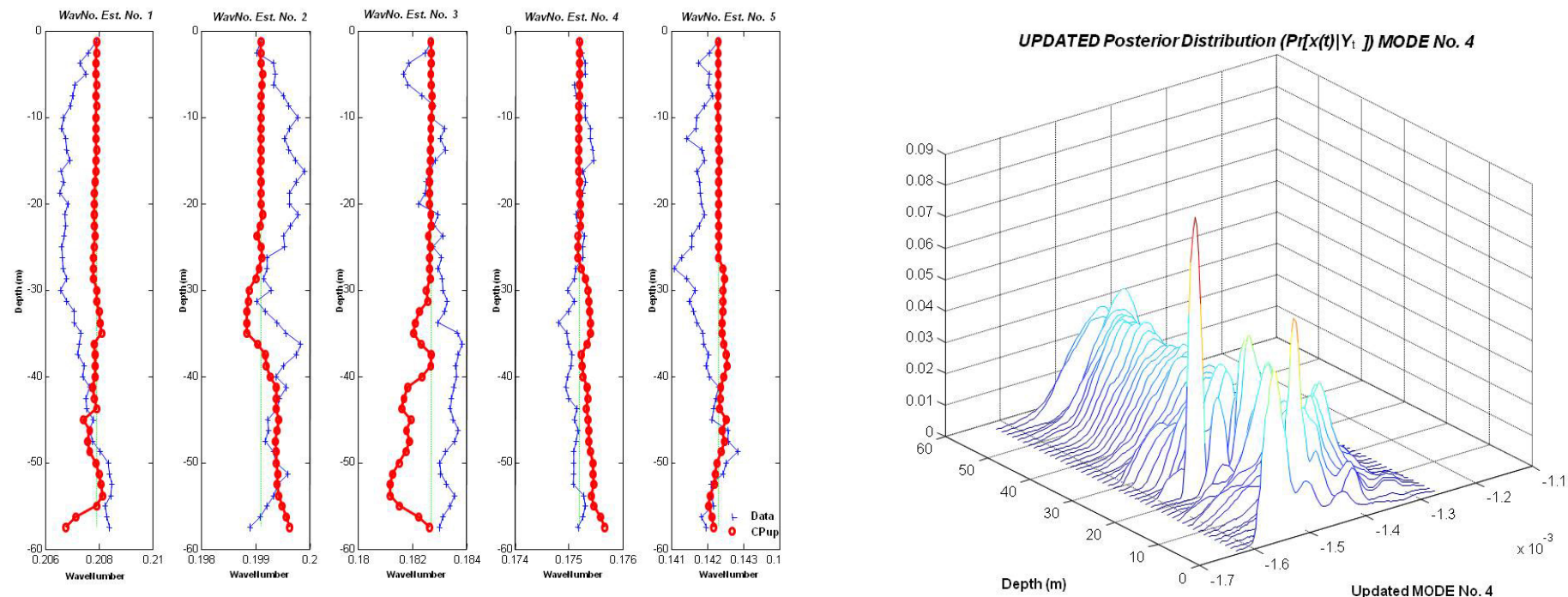

Fig. 6. Adaptive wavenumber parameter estimates from the Hudson Canyon 46-element array simulation using the MAP (red) particle filter.

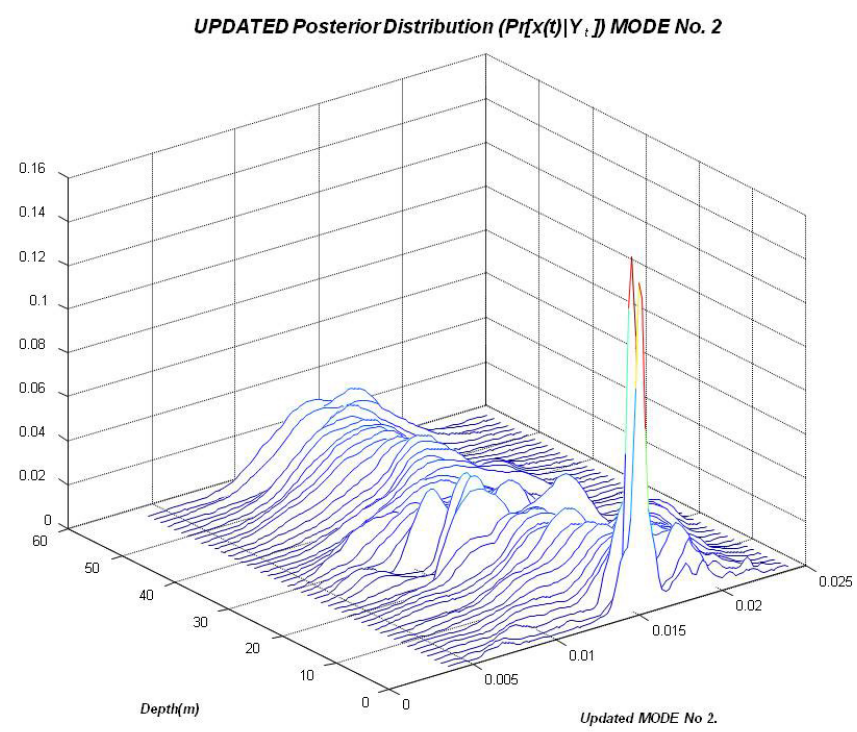

Fig. 7. PMF posterior estimation (mode 2) surface for synthesized Hudson Canyon 46-element array data (particle vs. time vs. probability).

in the posterior estimates. The wavenumber $P D F$ estimate corresponding to corresponding to mode 5 is shown in Fig. 9. Again we note the multiple, well-defined peaks in the posterior distribution leading to the MAP parameter estimate.

The pressure-field posterior dual peaks over the span of the water column. Visualizing a peak at each depth produces a "smooth" estimate (MAP) as shown in Fig. 10. This completes the analysis of the synthesized Hudson Canyon experiment and the $P F$ processing performance.

\section{SUMMARY}

This paper has discussed the development of an environmentally adaptive processor capable of tracking modes and

Fig. 8. PMF posterior estimation (mode 4) surface for synthesized Hudson Canyon 46-element array data (particle vs. time vs. probability).

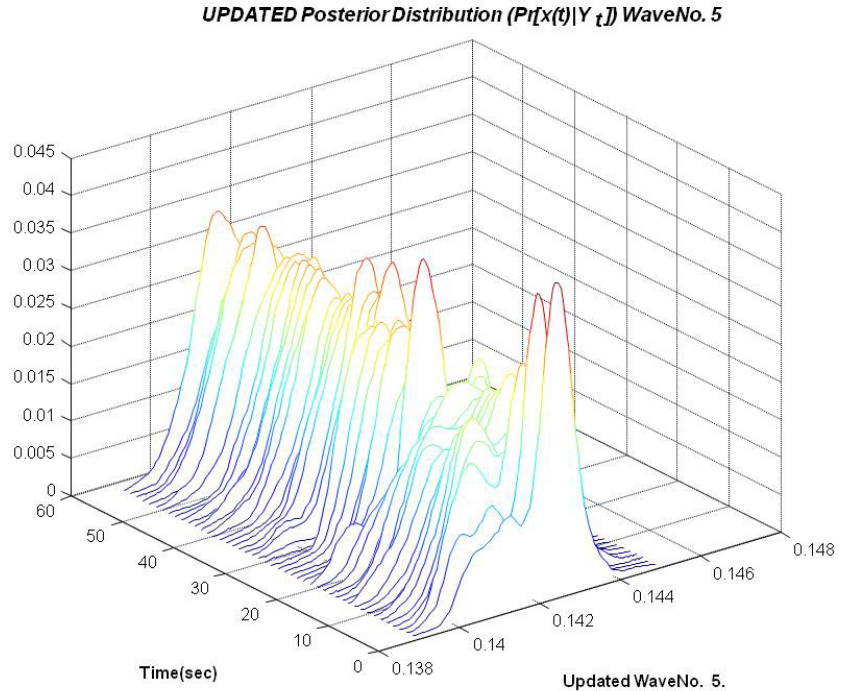

Fig. 9. PMF posterior estimation (wavenumber 5) surface for synthesized Hudson Canyon 46-element array data (particle vs. time vs. probability).

enhancing the raw pressure-field measurements obtained from a vertical hydrophone array in shallow water. The parametric adaption was based on simultaneously estimating the horizontal wavenumbers along with the modes and pressure-field as compared to previous work that concentrated on estimating the modal coefficients as the environmental parameters of interest [25], [26]. These parameters were more challenging from a processor design perspective because of their increased sensitivity to environmental change compared to the modal coefficients. We chose a Bayesian sequential design because of the varying nature of the shallow ocean and applied a normalmode model in state-space form to create a forward propagator. 


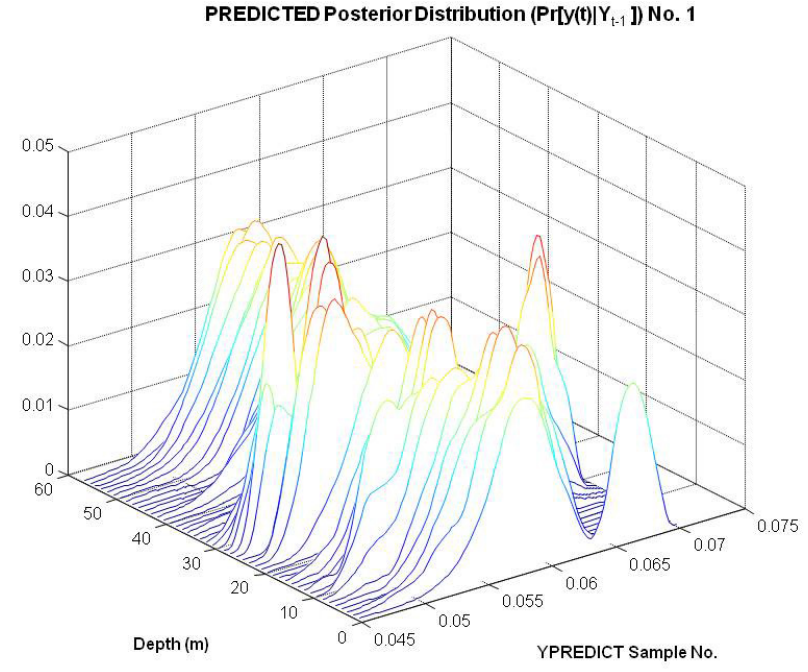

Fig. 10. Pressure-field posterior PMF estimation surface for synthesized Hudson Canyon data (particle vs. time vs. probability).

The algorithms applied were the unscented Kalman filter and the particle filter both modern approaches applied to this problem. We compared their performance and found slightly better results of the $P F$ over a 100-member ensemble. Our future efforts will be focused on extending the processors to actual measurement data.

\section{Acknowledgments}

This work performed under the auspices of the U.S. Department of Energy by Lawrence Livermore National Laboratory under Contract DE-AC52-07NA27344.

\section{REFERENCES}

[1] M. J. Hinich, "Maximum likelihood signal processing for a vertical array," J. Acoust. Soc. Am., 54, 499-503, 1973.

[2] C. S. Clay, "Use of arrays for acoustic transmission in a noisy ocean," Res. Geophys., 4, (4), 475-507, 1966.

[3] H. P. Bucker, "Use of calculated sound fields and matched-field detection to locate sound in shallow water," J. Acoust. Soc. Am., 59, 329-337, 1976.

[4] A. M. Richardson, and L. W. Note, "A posteriori probability source localization in an uncertain sound speed, deep ocean environment," $J$. Acoust. Soc. Am., 89, (6), 2280-2284, 1991.

[5] E. J. Sullivan and D. Middleton, "Estimation and detection issues in matched-field processing", IEEE J. Oceanic Eng., 18, (3), 156-167, 1993.

[6] F. B. Jensen and M. C. Ferla, "SNAP: the SACLANTCEN normal-model propagation model," Report SM-121, Italy: SACLANTCEN, 1979.

[7] M. B. Porter, "The KRACKEN normal mode program," Report SM-245, Italy: SACLANTCEN, 1991.

[8] H. Schmidt, "SAFARI: Seismo-acoustic fast field algorithm for range independent environments," Report SM-245, Italy: SACLANTCEN, 1987.

[9] F. B. Jensen, Kuperman, W. A., Porter M. B.,and H. Schmidt, Computational Ocean Acoustics New York: Amer. Instit. Physics Press, 1994.

[10] J. V. Candy and E.J. Sullivan. "Model-based processor design for a shallow water ocean acoustic experiment", J. Acoust. Soc. Am., 95, (4) 2038-2051, 1994.

[11] W. M. Carey, J. Doutt, R. Evans and L. Dillman, "Shallow water transmission measurements taken on the New Jersey continental shelf," IEEE J. Oceanic Eng., 20, (4), 321-336, 1995.
[12] A. R. Rogers, Y. Yamamoto and W. Carey, "Experimental investigation of sediment effect on acoustic wave propagation in shallow water," $J$. Acoust. Soc. Am., 93,1747-1761, 1993.

[13] C. S. Clay, and H. Medwin, Acoustical Oceanography. New York:Wiley, 1977.

[14] J. V. Candy and E. J. Sullivan. "Ocean acoustic signal processing: a model-based approach." J. Acoust. Soc. Am., 92, (12), 3185-3201, 1992.

[15] J. V. Candy and E. J. Sullivan. "Model-based identification: an adaptive approach to ocean-acoustic processing." IEEE Trans. Ocean. Engr., Vol. 21, No. 3, 273-289, 1996.

[16] A. Jazwinski, Stochastic Processes and Filtering Theory. New York:Academic Press, 1970.

[17] S. Julier and J. Uhlmann, "Unscented filtering and nonlinear estimation." Proc. IEEE, Vol. 92, No. 3, 401-422, 2000.

[18] J. V. Candy, Model-Based Signal Processing. Hoboken, N.J.:Wiley/IEEE Press, 2006.

[19] B. Ristic, S. Arulampalam and N. Gordon, Beyond the Kalman Filter: Particle Filters for Tracking Applications, Boston: Artech House, 2004

[20] O. Cappe, E. Moulines and T. Ryden, Inference in Hidden Markov Models, New York: Springer-Verlag, 2005.

[21] S. Godsill and P. Djuric, "Special Issue: Monte Carlo methods for statistical signal processing." IEEE Trans. Signal Proc., vol. 50, 2002.

[22] P. Djuric, J. Kotecha, J. Zhang, Y. Huang, T. Ghirmai, M. Bugallo and J. Miguez, "Particle Filtering," IEEE Signal Proc. Mag. vol. 20, No. 5, 19-38, 2003.

[23] S. Haykin and N. de Freitas, "Special Issue: Sequential state estimation: from Kalman filters to particle filters." Proc. IEEE, vol. 92, No. 3, 399574, 2004.

[24] J. V. Candy, Bayesian Signal Processing: Classical, Modern and Particle Filtering Methods. Hoboken, N.J.:Wiley/IEEE Press, 2009.

[25] J. V. Candy, "Adaptive particle filtering for mode tracking: a shallow ocean environment," Proceed. OCEANS'11, Santander, Spain, 2011.

[26] J. V. Candy, "An adaptive particle filtering approach to tracking modes is a varying shallow ocean environment," Proceed. OCEANS'11, Hawaii (Big Island), Hawaii, 2011.

\section{APPENDIX}

In this section we briefly develop the processors for our problem with details available in [24]. The basic adaptive problem we pursue in this paper can now be defined in terms of our mathematical models as:

GIVEN a set of noisy pressure-field and sound speed measurements varying in depth, $\left[\left\{p\left(r_{s}, z_{\ell}\right)\right\},\left\{c\left(z_{\ell}\right)\right\}\right]$ along with the underlying state-space model of Eqs. 17 and 18 with unknown wavenumbers, FIND the "best" (minimum error variance) estimate of the modal functions, that is, $\left\{\hat{\phi}_{m}\left(z_{\ell} \mid z_{\ell}\right)\right\},\left\{\hat{\theta}_{m}\left(z_{\ell} \mid z_{\ell}\right)\right\} ; m=1, \cdots, M$ and measurements (enhanced) $\left\{\hat{p}\left(r_{s}, z_{\ell}\right)\right\}$.

Before we attempt to describe the "new approaches" to the estimation problem, let us put these techniques in perspective. The $U K F$ is an alternative to the nonlinear or extended Kalman filter processor applied successfully in many of the modelbased ocean acoustic applications [1]-[14]. Like the EKF it is still restricted to a unimodal distribution (single peak), but that distribution need not be Gaussian. It also performs a linearization (statistical), but not of the system dynamical model, but of an inherent nonlinear vector transformation requiring "sigma points" which deterministically characterize the underlying unimodal distribution. These points have been pre-calculated for the Gaussian case [17], [18]. It has been shown that the UKF clearly outperforms the EKF and its variants (iterated $E K F$, higher order EKFs, etc.). and is more 


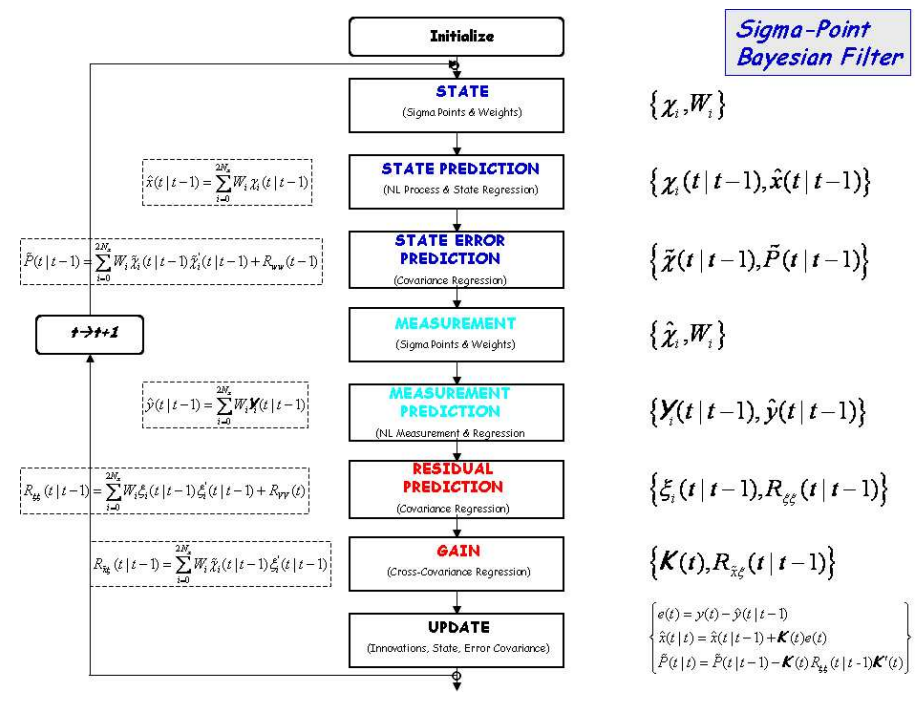

Fig. 11. Unscented Kalman filter algorithm flow diagram: initialization, prediction, update and innovation with $t$ the index variable.

accurate and precise besides being much easier to implement, since Jacobians are no longer required. Note also that if we place the EKF/UKF into the Bayesian framework to follow, then we see that the underlying posterior distribution has already been decided to be approximately multivariate Gaussian with the objective to extract the corresponding conditional mean and covariance as accurately as possible. Therefore, we see that the $U K F$ provides the multivariate posterior solution

$$
\begin{aligned}
& \hat{p}\left[\phi\left(z_{\ell}\right) \mid P_{z}\right] \approx(2 \pi)^{N_{x} / 2}\left|R_{\phi \phi}\left(z_{\ell} \mid z_{\ell}\right)\right|^{-1 / 2} \times \exp \left\{-\frac{1}{2}\right. \\
& \left.\left(\phi\left(z_{\ell}\right)-\hat{\phi}\left(z_{\ell} \mid z_{\ell}\right)\right)^{T} R_{\phi \phi}^{-1}\left(z_{\ell} \mid z_{\ell}\right)\left(\phi\left(z_{\ell}\right)-\hat{\phi}\left(z_{\ell} \mid z_{\ell}\right)\right)\right\}
\end{aligned}
$$

where $\hat{\phi}\left(z_{\ell} \mid z_{\ell}\right)$ is the conditional modal mean at depth $z_{\ell}$ and $R_{\phi \phi}\left(z_{\ell} \mid z_{\ell}\right)$ is the conditional modal covariance based on pressure-field measurements up to depth $z_{\ell}$.

A detailed flow diagram of the $U K F$ is shown in Fig. 11 where we note the basic predictor/update structure. Much of the algorithm is devoted to the statistical linearization in which regression estimators are used to perform the transformation while the usual Kalman filtering equations are used to perform the updates. We refer the interested reader to the current texts or basic papers for more details [19]-[24].

Next we consider the particle filter processor. A particle filter is a different approach to nonlinear filtering in that it removes the restriction of additive Gaussian noise sources and is clearly capable of characterizing multimodal distributions. In fact, it might be easier to think of the $P F$ as a histogram or kernel density like estimator in the sense that it is an empirical probability mass function $(P M F)$ that approximates the desired posterior distribution such that statistical inferences can easily be performed and statistics extracted directly. The computational burden of the $P F$ is much higher than that of the KF, since it must provide an estimate of the underlying state posterior distribution component-by-component at each $z_{\ell}$-step along with the fact that the number of samples to characterize the distribution is equal to the number of particles.

$$
\hat{\operatorname{Pr}}\left[\phi\left(z_{\ell}\right) \mid P_{z}\right]=\sum_{i=1}^{N_{p}} \mathcal{W}_{i}\left(z_{\ell}\right) \delta\left(\phi\left(z_{\ell}\right)-\phi_{i}\left(z_{\ell}\right)\right) \quad \forall z_{\ell}
$$

$$
\begin{array}{cl}
W_{i}\left(z_{\ell}\right) & \propto \hat{\operatorname{Pr}}\left[\phi_{i}\left(z_{\ell}\right) \mid P_{z}\right] \text { is the estimated weights at } z_{\ell} ; \\
\phi_{i}\left(z_{\ell}\right) & \text { is the } i \text {-th particle at depth } z_{\ell} ; \\
\hat{\operatorname{Pr}}[\cdot] & \text { is the estimated empirical posterior distribution; } \\
P_{z} & \text { is the set of batch pressure-field measurements, } \\
& P_{z}=\left\{p\left(r_{s}, z_{1}\right) \cdots p\left(r_{s}, z_{L}\right)\right\}
\end{array}
$$

Thus, we see that once the underlying posterior is available, the estimates of important statistics can be extracted directly. For instance, the maximum a posteriori (MAP) estimate is simply found by locating a particular particle $\hat{\phi}_{i}\left(z_{\ell}\right)$ corresponding to the maximum of the $P M F$, while the conditional mean or equivalently the minimum mean-squared error (MMSE) estimate is calculated by integrating the posterior [24].

There are a variety of $P F$ algorithms available, but perhaps the simplest is the bootstrap technique [24] which we apply to our problem (see Fig. 12). The PF design for our problem using the bootstrap approach requires the conditional state transition probability, $\operatorname{Pr}\left[\Phi\left(z_{\ell}\right) \mid \Phi\left(z_{\ell-1}\right)\right]$, and the likelihood (probability) $\operatorname{Pr}\left[p\left(r_{s}, z_{\ell}\right) \mid \Phi\left(z_{\ell}\right)\right]$. Here the state transition is characterized by the underlying augmented statespace model for each mode. For the bootstrap implementation, we need only draw noise samples from the state and parameter distributions and use the dynamic models above (normalmode/random walk) to generate the set of particles, $\left\{\Phi_{m i}\left(z_{\ell}\right)\right\}$ for each $i=1, \cdots, N_{p}$.

The likelihood, on the other hand, is determined from the nonlinear pressure-field measurement model of Eq. 18, that is, for each mode we have

$p_{m i}\left(r_{s}, z_{\ell}\right):=\sum_{m=1}^{M} \beta_{m}\left(r_{s}, z_{s} ; \theta_{m}\left(z_{\ell}\right)\right) \phi_{m}\left(z_{\ell}\right)+v\left(z_{\ell}\right) ; \ell=1, \cdots, L$

with

$$
\beta_{m}\left(r_{s}, z_{s}\right):=q H_{0}\left(\theta_{m}\left(z_{\ell}\right) r_{s}\right) \phi_{m}\left(z_{s}\right)
$$

and therefore the scalar likelihood (assuming Gaussian noise) is

$$
\begin{aligned}
& \operatorname{Pr}\left[p\left(r_{s}, z_{\ell}\right) \mid \Phi\left(z_{\ell}\right)\right]=\frac{1}{\sqrt{2 \pi R_{v v}}} \times \exp \left\{-\frac{1}{2 R_{v v}}\right. \\
& \left.\left(p\left(r_{s}, z_{\ell}\right)-\sum_{m=1}^{M} \beta_{m}\left(r_{s}, z_{s} ; \theta_{m}\left(z_{\ell}\right)\right) \phi_{m}\left(z_{\ell}\right)+v\left(z_{\ell}\right)\right)^{2}\right\}
\end{aligned}
$$

Thus, we estimate the posterior distribution using a sequential Monte Carlo approach and construct a bootstrap particle filter [19]-[24] using the following steps: 


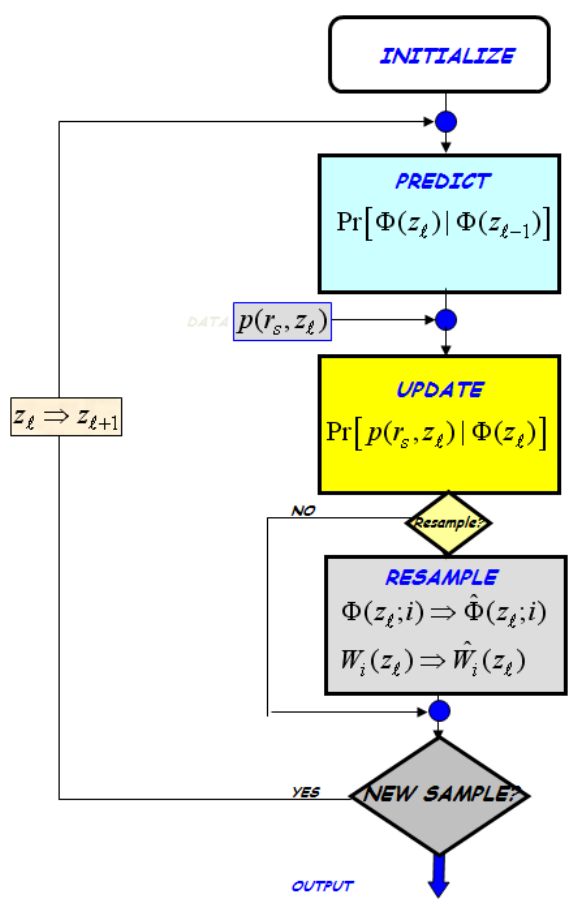

Fig. 12. Bootstrap particle filter algorithm flow diagram: prediction, update and resampling with $z_{\ell}$ the index variable.

- Initialize: $\Phi_{m}(0), w_{z_{\ell}} \sim \mathcal{N}\left(0, R_{w w}\right), W_{i}(0)=1 / N_{p} ; i=$ $1, \cdots, N_{p}$

- $\underline{\text { State Transition: }} \Phi_{m}\left(z_{\ell}\right)=\mathbf{A}_{m}\left(z_{\ell-1}\right) \Phi_{m}\left(z_{\ell-1}\right)+$ $\mathbf{w}_{m}\left(z_{\ell-1}\right)$

- Likelihood Probability: $\operatorname{Pr}\left[p\left(r_{s}, z_{\ell}\right) \mid \Phi\left(z_{\ell}\right)\right]$;

- Weights: $W_{i}\left(z_{\ell}\right)=W_{i}\left(z_{\ell-1}\right) \times \operatorname{Pr}\left[\Phi_{m}\left(z_{\ell}\right) \mid \Phi_{m}\left(z_{\ell-1}\right)\right]$;

- $\underline{\text { Normalize: }} \mathcal{W}_{i}\left(z_{\ell}\right)=\frac{W_{i}\left(z_{\ell}\right)}{\sum_{i=1}^{N_{p}} W_{i}\left(z_{\ell}\right)}$;

- Resample: $\tilde{\Phi}_{i}\left(z_{\ell}\right) \Rightarrow \Phi_{i}\left(z_{\ell}\right)$;

- Posterior: $\hat{\operatorname{Pr}}\left[\Phi_{m}\left(z_{\ell}\right) \mid P_{z}\right]=\sum_{i=1}^{N_{p}} \mathcal{W}_{i}\left(z_{\ell}\right) \delta\left(\phi\left(z_{\ell}\right)-\right.$ $\left.\phi_{i}\left(z_{\ell}\right)\right)$; and

- MAP Estimate: $\hat{\Phi}_{i}^{M A P}(z)=\max _{i} \hat{\operatorname{Pr}}\left[\phi_{i}\left(z_{\ell}\right) \mid P_{z}\right]$;

- MMSE Estimate: $\hat{\Phi}_{i}^{M M S E}(z)=\frac{1}{N_{p}} \sum_{i=1}^{N_{p}} \mathcal{W}_{i}\left(z_{\ell}\right) \phi_{i}\left(z_{\ell}\right)$

More details can be found in the referenced textbooks and papers [19]-[24]. Thus, we see that there exists a fundamental philosophical difference between the UKF (Kalman) processor and the $P F$ processor. Their implementations are completely different as well: one based on approximating the required distribution through statistical linearization and one through an empirical PMF estimator. This completes the Appendix. 